\title{
DIGITALCOMMONS
}

$@$ WAYNESTATE-

Wayne State University

10-1-2006

\section{Multiobjective Optimization Problems with Equilibrium Constraints}

Boris S. Mordukhovich

WayneState University, boris@math.wayne.edu

\section{Recommended Citation}

Mordukhovich, Boris S., "Multiobjective Optimization Problems with Equilibrium Constraints" (2006). Mathematics Research Reports. Paper 40.

http://digitalcommons.wayne.edu/math_reports/40

This Technical Report is brought to you for free and open access by the Mathematics at DigitalCommons@WayneState. It has been accepted for inclusion in Mathematics Research Reports by an authorized administrator of DigitalCommons@WayneState. 


\title{
MULTIOBJECTIVE OPTIMIZATION PROBLEMS WITH EQUILIBRIUM CONSTRAINTS
}

BORIS S. MORDUKHOVICH

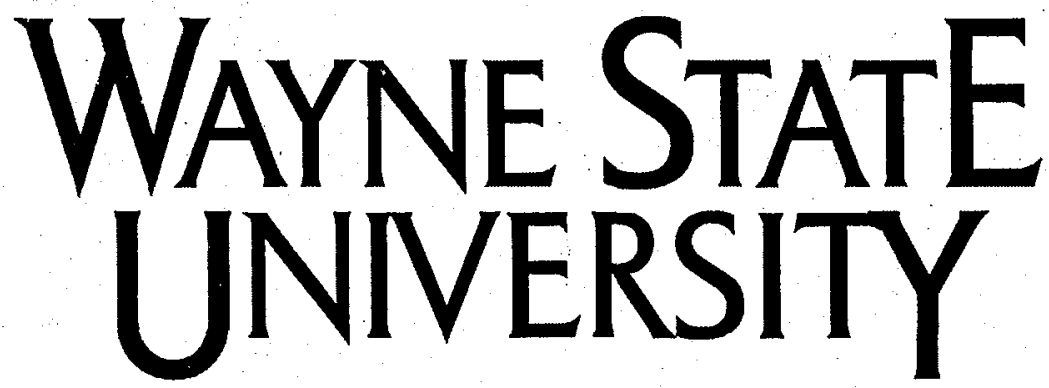

Detroit, MI 48202

Department of Mathematics

Research Report

\author{
2006 Series \\ \#10
}

This research was partly supported by the National Science Foundation and the Australian Research Council 


\title{
MULTIOBJECTIVE OPTIMIZATION PROBLEMS WITH EQUILIBRIUM CONSTRAINTS ${ }^{1}$
}

\author{
"'BÓRIS S. MORDUKHOVICH ${ }^{2}$ \\ Dedicated to Steve Robinson in honor of his 65th birthday
}

\begin{abstract}
The paper is devoted to new applications of advanced tools of modern variational analysis and generalized differentiation to the study of broad classes of multiobjective optimization problems subject to equilibrium constraints in both finite-dimensional and infinite-dimensional settings. Performance criteria in multiobjective/vector optimization are defined by general preference relationships satisfying natural requirements, while equilibrium constraints are described by parameterized generalized equations/variational conditions in the sense of Robinson. Such problems are intrinsically nonsmooth and are handled in this paper via appropriate normal/coderivative/subdifferential constructions that exhibit full calculi. Most of the results obtained are new even in finite dimensions, while the case of infinite-dimensional spaces is significantly more involved requiring in addition certain "sequential normal compactness" properties of sets and mappings that are preserved under a broad spectrum of operations.
\end{abstract}

Key words.- multiobjective optimization-preference relationships-equilibrium-constraints-variational analysis-generalized differentiation-necessary optimality conditions

Mathematics Subject-Glassification-(2000):-90C29,-90C30,49J52, 49J53, 49K27

\section{Introduction}

This paper concerns the study of multiobjective optimization problems with equilibrium constraints (abrr. MOPECs) described by general preference relations subject to constraints given in the form

$$
0 \in q(x, y)+Q(x, y)
$$

where $q: X \times Y \rightarrow P$ is a single-valued mapping while $Q: X \times Y \Rightarrow P$ is a set-valued mapping between the corresponding Banach spaces, $y \in Y$ stands for the decision variable, and $x \in X$ is a parameter.

Models of type (1.1) were introduced by Robinson [22] in the end of 1970s, and since that time they have played a crucial role in many aspects of optimization and variational analysis. It seems that the original motivation for Robinson was to describe variational inequalities and complementarity problems in the form of "generalized equations," which are distinguished from standard equations by the presence of the multivalued term $Q$ while

\footnotetext{
${ }^{1}$ Research was partially supported by the National Science Foundation under grants DMS-0304989 and DMS-0603846 and by the Australian Research Council under grant DP-0451168.

${ }^{2}$ Department of Mathematics, Wayne State University, Detroit, Michigan 48202, USA; boris@math.wayne.edu
} 
allowing one to explore this similarity for their qualitative study and numerical solution. Indeed, generalized equations (1.1) reduce to the parametric variational inequalities

$$
\text { find } y \in \Omega \text { with }\langle q(x, y), v-y\rangle \geq 0 \text { for all } v \in \Omega
$$

when $Q(y)=N(y ; \Omega)$ in $(1.1)$ is the classical normal cone mapping to a convex set $\Omega$. Based on formalism (1.1), Robinson and his followers developed strong results in sensitivity analysis and numerical methods of solving variational inequalities, complementarity and optimization problems, etc.; see particularly the seminal papers by Robinson [22, 23], his recent survey [24], and the fundamental two-volume monograph by Facchinei and Pang [6].

It has been well realized that constraints (1.1) can describe certain equilibrium conditions, in particular, those arising from the solution of lower-level parametric problems in hierarchical optimization (e.g., in bilevel programming). On this basis, minimization problems subject to constraints of type (1.1), which express sets of feasible solutions to the upper level of hierarchical optimization, are called mathematical programs with equilibrium constraints (MPECs); see the books by Luo, Pang, and Ralph [10] and by Outrata, Kočvara and Zowe [20] for various approaches and results for such problems; more recent extensive bibliographies and commentaries on MPECs can be found in $[3,6,13]$.

The main goal of this paper is to study multiobjective optimization problems subject to constraints of generalized equation type (1.1) and their important specifications. Problems of this kind have been considered in finite-dimensional spaces by Ye and Zhu [27], where the upper-level optimality are defined in terms of certain "regular" preference relations and equilibrium constraints are given via variational inequalities (1.2). They have also been partly studied in finite dimensions in the author's paper [11] devoted to MOPECs with preference relations on the upper level given via "generalized order optimality" that extends various Pareto-like efficiency/equilibrium notions. The recent monograph [13] carefully develops necessary optimality conditions for multiobjective problems of the latter type to the case of infinite-dimensional spaces. Observe that such problems can be treated as a kind of equilibrium problems with equilibrium constraints (EPECs), where certain equilibrium relations are presented on both lower and upper levels of hierarchy; we refer the reader to $[7,8,19,15]$ for other EPEC concepts, developments, discussions, and applications.

This paper is mainly devoted to deriving new qualified necessary optimality conditions for broad classes of MOPECs in finite-dimensional and infinite-dimensional spaces, where the notions of multiobjective/vector optimality on the upper level are defined via general preference relations satisfying certain natural requirements. We employ advanced tools of variational analysis and generalized differentiation to obtain such conditions in general MOPEC frameworks and in more specific settings important for applications. Note that our techniques, revolving around the extremal principle of variational analysis and welldeveloped generalized differential calculus for the dual-space/coderivative-like constructions [12], allow us avoid certain conventional troubles in the study of optimization problems with equilibrium constraints (e.g., those related to the failure of the Mangasarian-Fromovitz and the like constraint qualifications) and to establish verifiable optimality conditions for the new classes of MOPECs under consideration in both finite and infinite dimensions.

The rest of the paper is organized as follows. Section 2 presents a brief necessary 
review of the basic generalized differential constructions of variational analysis and normal compactness properties needed for formulations and proofs of the main results.

In Section 3 we formulate and study multiobjective problems of parametric optimization with preference relations satisfying appropriate requirements and general constraints of the type $y \in S(x)$. We derive qualified necessary optimality conditions for such problems and present their specification' in the cases of constraints systems $S(\cdot)$ described by finitely many equalities and inequalities and by solution maps to the generalized equations (1.1), where both single-valued part $q(x, y)$ and set-valued part $Q(x, y)$ are parameter-dependent.

Section 4 is devoted to the detailed study of MOPECs with general preference relations and the multivalued part $Q(x, \ddot{y})$ of the equilibrium constraints (1.1) given in the so-called composite subdifferential form

$$
Q(x, y)=\partial(\psi \circ g)(x, y)
$$

where $g$ is a single-valued mapping between Banach spaces and $\psi$ is an extended-realvalued function. The subdifferential structure (1.3) with composite potentials (mechanical terminology) is typical for many applications related to parametric optimization (on the level level) and variational (hemivariational, quasivariational) inequalities.

In the final Section 5 we consider special MOPECs with another subdifferential structure of equilibrium constraints with the multivalued part $Q(x, y)$ given in the composite form

$$
Q(x, y)=(\partial \psi \circ g)(x, y)
$$

called the composite subdifferential field of the generalized equation (1.1). Structure (1.4) is useful, e.g., for describing equilibrium constraints governed by implicit complementarity conditions; see below. The results obtained in Sections 4 and 5 are based on the necessary optimality conditions established for general MOPECs in Section 3 and on the second-order subdifferential calculus developed in [12].

Our notation is basically standard; see $[12,13]$. Recall that, given a set-valued mapping $F: X \rightrightarrows X^{*}$ between a Banach space $X$ and its topological dual $X^{*}$, the sequential PainlevéKuratowski upper/outer limit of $F$ as $x \rightarrow \bar{x}$ with respect to the norm topology of $X$ and the weak* topology $w^{*}$ of $X^{*}$ is

$$
\begin{aligned}
\underset{x \rightarrow \bar{x}}{\operatorname{Limsup}} F(x):=\left\{x^{*} \in X^{*} \mid\right. & \exists \text { sequences } x_{k} \rightarrow \dot{\bar{x}} \text { and } x_{k}^{*} \stackrel{w^{*}}{\rightarrow} x^{*} \\
& \text { with } \left.x_{k}^{*} \in F\left(x_{k}\right) \text { for all } k \in \mathbb{N}\right\},
\end{aligned}
$$

where $\mathbb{I N}:=\{1,2, \ldots\}$. Recall also that the symbols $x \stackrel{\Omega}{\rightarrow} \bar{x}$ and $x \stackrel{\varphi}{\rightarrow} \bar{x}$ signify, respectively, that $x \rightarrow \bar{x}$ with $x \in \Omega$ and that $x \rightarrow \bar{x}$ with $\varphi(x) \rightarrow \varphi(\bar{x})$ for sets $\Omega \subset X$ and extendedreal-valued functions $\varphi: X \rightarrow \overline{\mathbb{R}}:=(-\infty, \infty]$. Unless otherwise stated, all the spaces under consideration are Banach with the norm $\|\cdot\|$ and the canonical pairing $\langle\cdot, \cdot\rangle$ between the space in question and its dual. We use $B_{X}$ to denote the closed unit ball of $X$, where the subindex " $X$ " is omitted when there is no confusion. 


\section{Tools of Variational Analysis}

We start with a brief necessary review of the basic generalized differential constructions of variational analysis and some of their properties widely used in what follows. This is taken from the author's book [12], where the reader can find a comprehensive theory for these constructions with extensive discussions, references, and commentaries.

In fact, most of the results obtained in this paper require a special Asplund structure of the spaces in question. Recall that a Banach space $X$ is $A$ splund if each of its separable subspace has a separable dual. This is a broad class of Banach spaces including all reflexive spaces and all spaces with separable duals; see, e.g., the book by Phelps [21] for more information, references, and discussions.

To simplify the exposition, we present in this section only those basic definitions and properties that hold in Asplund spaces, while their more general versions and modifications will be given in the subsequent sections where they are actually needed; anyway, the reader can find all the details in the book [12].

Starting with generalized normals to sets, take $\Omega \subset X$ and $\bar{x} \in \Omega$ and define the (basic, limiting) normal cone to $\Omega \subset X$ at $\bar{x}$ by

$$
N(\bar{x} ; \Omega):=\underset{x \rightarrow \bar{x}}{\operatorname{Limsup}} \widehat{N}(x ; \Omega),
$$

where $\widehat{N}(x ; \Omega)$ stands for the prenormal, or the Fréchet normal, cone to $\Omega$ at $x$ given by

$$
\widehat{N}(x ; \Omega):=\left\{x^{*} \in X^{*} \mid \underset{\substack{\Omega \rightarrow x \\ \limsup _{\Omega}}}{\left\langle x^{*}, u-x\right\rangle} \| 0\right\} \text { whenever } x \in \Omega
$$

and $\widehat{N}(x ; \Omega):=\emptyset$ if $x \notin \Omega$. Note that the basic normal cone $N(\bar{x} ; \Omega)$ is often nonconvex, in contrast to $\widehat{N}(\bar{x} ; \Omega)$, which may be empty at boundary points. In particular,

$$
N\left(0 ; \text { epi }(-|x|)=\left\{(u, v) \in \mathbb{R}^{2}|v=-| u \mid\right\} \text { while } \widehat{N}(0 ; \operatorname{epi}(-|x|)=\emptyset\right.
$$

where epi $\varphi$ stands for the standard epigraphical set of the function. Nevertheless, the basic normal cone $N(\cdot ; \Omega)$ enjoys extended calculus rules ("full calculus"), which are much better not only in comparison with $\widehat{N}(; \Omega)$ but also with the convex closure of $N(\cdot ; \Omega)$ that agrees with the normal cone by Clarke; see $[12,25]$ for more discussions and references.

Given a set-valued mapping $F: X \rightrightarrows Y$ and a point $(\bar{x}, \bar{y})$ from its graph

$$
\operatorname{gph} F:=\{(x, y) \in X \times Y \mid y \in F(x)\},
$$

define the coderivative $D^{*} F(\bar{x}, \bar{y}): Y^{*} \rightrightarrows X^{*}$ of $F$ at $(\bar{x}, \bar{y})$ by

$$
D^{*} F(\bar{x}, \bar{y})\left(\bar{y}^{*}\right):=\left\{x^{*} \in X^{*} \mid\left(x^{*},-y^{*}\right) \in N((\bar{x}, \bar{y}) ; \operatorname{gph} F)\right\}, \quad \bar{y}^{*} \in Y^{*},
$$

where $\bar{y}$ is omitted when $F=f: X \rightarrow Y$ is single-valued. If in the latter case $f$ is strictly differentiable at $\bar{x}$ (which is automatic when $f$ is $C^{1}$ around this point), then

$$
D^{*} f(\bar{x})\left(y^{*}\right)=\left\{\nabla f(\bar{x})^{*} y^{*}\right\}, \quad y^{*} \in Y^{*},
$$


via the adjoint derivative operator $\nabla f(\bar{x})^{*}: Y^{*} \rightarrow X^{*}$. In $[12,13]$, the reader can find equivalent analytic representations of the coderivative and its efficient calculations for various classes of nonsmooth single-valued and set-valued mappings.

Let $\varphi: X \rightarrow \overline{\mathbb{R}}$ be an extended-real-valued function finite at $\bar{x}$ : Then

$$
\partial \varphi(\bar{x}):=\operatorname{Limsup}_{x \underline{\varphi} \underline{x}_{\bar{x}}} \widehat{\partial} \varphi(x)
$$

is the (basic, limiting) subdifferential of $\varphi$ at $\bar{x}$, where

$$
\widehat{\partial} \varphi(x):=\left\{x^{*} \in X^{*} \mid \frac{\varphi(u)-\varphi(x)-\left\langle x^{*}, u-x\right\rangle}{\|u-x\|} \geq 0\right\}
$$

is the presubdifferential of $\varphi$ at $x$ known also as Fréchet, regular, viscosity subdifferential of $\varphi$ at $x$. Observe the useful geometric descriptions of the subdifferential (2.4) via the basic normal cone and coderivative:

$$
\partial \varphi(\bar{x})=\left\{x^{*} \in X^{*} \mid\left(x^{*},-1\right) \in N((\bar{x}, \varphi(\bar{x})) ; \operatorname{epi} \varphi)\right\}=D^{*} E_{\varphi}(\bar{x}, \varphi(\bar{x}))(1),
$$

where $E_{\varphi}: X \rightrightarrows \mathbb{R}$ stands for the epigraphical multifunction associated with the function $\varphi$ by $\operatorname{gph} E=\operatorname{epi} \varphi$. On the other hand, the geometrically defined coderivative (2.3) admits, in the case of single-valued mappings $f: X \rightarrow Y$, the convenient subdifferential representation via (2.4) known as the scalarization formula:

$$
D^{*} f(\bar{x})\left(y^{*}\right)=\partial\left\langle y^{*}, f\right\rangle(\bar{x}), \quad y^{*} \in Y^{*},
$$

provided that the mapping $f$ is strictly Lipschitzian at $\bar{x}$, i.e., it is Lipschitz continuous around this point and the sequence

$$
\left\{\frac{f\left(x_{k}+t_{k} v\right)-f\left(x_{k}\right)}{t_{k}}\right\}, \quad k \in \mathbb{N}
$$

contains a norm convergent subsequence whenever $x_{k} \rightarrow \bar{x}$ and $v$ belongs to some neighborhood of the origin. The latter requirement is obviously redundant if $\operatorname{dim} Y<\infty$; see [12, Subsection 3.1.3] for characterizations, verifiable sufficient conditions, and applications of the strict Lipschitzian property in the case of infinite-dimensional spaces $Y$.

As follows from the definitions and the example above, our basic coderivative and subdifferential constructions (2.3) and (2.4) may have nonconvex values even in very simple situations; in particular, we have $\partial(-|x|)(0)=\{-1,1\}$. It seems surprising therefore, from the viewpoint of conventional techniques in convex analysis totally based on separation theorems, that they enjoy full calculus. The main driving force for this calculus and many other results of variational analysis is the fundamental extremal principle (see [12, Chapter 2] for the detailed study and discussions), which is a variational counterpart of the classical convex separation in nonconvex settings. Note that the convexification operation for the limiting normals discussed above happens to be especially unwelcome for the case of graphically Lipschitzian sets, when it often gives the whole space (always a linear subspace of the maximum dimension), and thus exclude any potential applications; see [12, Subsection 1.2.2 and Subsection 3.2.4] for exact formulations and more details. In particular,

$$
N(0 ; \operatorname{gph}|x|)=(\operatorname{gph}|u|) \cup\left\{(u, v) \in \mathbb{R}^{2}|v \leq-| u \mid\right\} \text { with } \operatorname{co} N(0 ; \operatorname{gph}|x|)=\mathbb{R}^{2} .
$$


It is important to observe that graphically Lipschitzian sets include not only graphs of Lipschitzian mappings but also those for monotone and subdifferential operators unavoidably encountered in variational inequalities, complementarity problems, etc. Furthermore, graphical sets appear in the very definition of coderivatives, which play a crucial role in our analysis of such and related problems particularly conducted in this paper.

For our main results here, we also need the following notion of generalized normals to parameterized (or moving) sets. Given $\Omega: Z \rightrightarrows X$ and $(\bar{z}, \bar{x}) \in \mathrm{gph} \Omega$, defined the extended normal cone to $\Omega(\bar{z})$ at $\bar{x}$ by

$$
N_{+}(\bar{x} ; \Omega(\bar{z})):=\operatorname{Limsup}_{(z, x) \rightarrow(\bar{z}, \bar{x})} \widehat{N}(x ; \Omega(z))
$$

via the Kuratowski-Painlevé outer limit (1.5) of prenormals (2.2) at points $(z, x) \in \operatorname{gph} \Omega$ nearby. We always have the inclusion.

$$
N(\bar{x} ; \Omega(\bar{z})) \subset N_{+}(\bar{x} ; \Omega(\bar{z})),
$$

where the equality holds under the so-called normal semicontinuity of $\Omega$ at $(\bar{z}, \bar{x})$, which is the case for a broad class of mappings under reasonable assumptions; see [13, Subsection 5.3.3] for more discussions and sufficient conditions. Note that, even in the absence of normal semicontinuity, the extended normal cone (2.7) enjoys comprehensive calculus rules similarly to the basic one $(2.1)$.

Finally in this section, recall some "normal compactness" properties needed in this paper that are automatic in finite dimensions while playing a crucial role in many aspects of infinite-dimensional variational analysis and its applications; see $[12,13]$ for more details. A set $\Omega \subset X$ locally closed around $\vec{x}$ is sequentially normally compact (SNC) at this point if for every sequences $x_{k} \stackrel{\Omega}{\rightarrow} \bar{x}$ and $x_{k}^{*} \in \widehat{N}\left(x_{k} ; \Omega\right)$ one has the implication

$$
x_{k}^{*} \stackrel{w^{*}}{\rightarrow} 0 \Longrightarrow\left\|x_{k}^{*}\right\| \rightarrow 0 \text { as } k \rightarrow \infty \text {. }
$$

This property always holds if $\Omega$ is compactly epi-Lipschitzian (CEL) around $\bar{x}$ in the sense of Borwein and Strójwas [2] although in general the implication CEL $\Rightarrow$ SNC is strict even for convex cones in (nonseparable) Asplund spaces; see [5] for a comprehensive study of the relationships between the SNC and CEL properties. Naturally, the SNC property of a mapping is induced by this property of its graph. Note that if $f: X \rightarrow Y$ is locally Lipschitzian around $\bar{x}$, it is always SNC at this point provided that $Y$ is finite-dimensional while $X$ is a general Banach space.

Considering a parameter-dependent set $\Omega(z)$, we say that it is imagely $S N C$ (or briefly ISNC) at $(\bar{z}, \bar{x}) \in \operatorname{gph} \Omega$ if for any sequences $\left(z_{k}, x_{k}, x_{k}^{*}\right)$ satisfying

$$
x_{k}^{*} \in \widehat{N}\left(x_{k} ; \Omega\left(z_{k}\right)\right), \quad\left(z_{k}, x_{k}\right) \stackrel{\operatorname{gph} \Omega}{\rightarrow}(\bar{z}, \bar{x}), \quad \text { and } \quad x_{k}^{*} \stackrel{w^{*}}{\rightarrow} 0
$$

one has $\left\|x_{k}^{*}\right\| \rightarrow 0$ as $k \rightarrow \infty$. Note that the ISNC property is obviously automatic in finite dimensions, while in infinite dimensions it holds under certain uniform Lipschitz-type assumptions; see the above reference and [17] for precise results and discussions.

The crucial fact for the theory and especially for applications of the afore-mentioned normal compactness properties consists of the validity for them the well-developed SNC cal culus [12] ensuring the preservation of these and related properties under various operations. This calculus is also based on the extremal principle of variational analysis. 


\section{Necessary Conditions in Multiobjective Optimization and General MOPECs}

In this section we establish necessary conditions for local optimal solutions to general problems of parametric multiobjective optimization and those with equilibrium constraints (1.1), where the notion of multiobjective/vector optimality on the upper level is defined by arbitrary preference relations satisfying the requirements formulated below.

Definition 3.1 (preferences). Let $Z$ be a topological space, let $\Xi \subset Z \times Z$, and let $\bar{z} \in Z$. Define a relation $\prec$ on $Z$ by

$$
z_{1} \prec z_{2} \text { if }\left(z_{1}, z_{2}\right) \in \Xi
$$

and say that $\prec$ is a PREFERENCE around $\bar{z}$ if there is a neighborhood $U$ such that:

(a) $(z, z) \notin \Xi$ for all $z \in U$;

(b) $z \in \operatorname{cl} \mathcal{L}(z)$ for all $z \in U$, where

$$
\mathcal{L}(z):=\{u \in Z \mid u \prec z\}
$$

(c) $v \prec z$ whenever $v \in \mathcal{L}(u), u \prec z$, and $v, z, u \in U$.

The broad class of preferences considered in Definition 3.1 includes the vast majority of particular preference relations used in vector optimization; see $[9,13,16]$ for more discussions, examples, and references. In what follows, we are going to study MOPECs whose objectives on the upper level are formalized via arbitrary preference relations satisfying properties (a)-(c).

Let us begin with a general class of multiobjective problems of parametric optimization, where constraints are defined by arbitrary set-valued mappings of closed graph given in the form $y \in S(x)$, with the decision variable $y \in Y$ and the parameter $x \in X$. In particular, the constraint mapping $S: X \Rightarrow Y$ can be described by finitely many equalities and inequalities as in nonlinear programming, in the form $g(x, y) \in \Theta$ covering problems of conic and semidefinite programming, in operator forms involving various kinds of operators (e.g., integral and differential) between infinite-dimensional spaces as in problems of optimal control, etc. The primary goal of this and subsequent sections is to study multiobjective problems of parametric optimization with constraint mappings given as parameterized sets of solutions (solution maps) to the generalized equations of type (1.1) and their remarkable specifications labeled as equilibrium constraints. As mentioned, such constraints on the upper level may arise as parameterized sets of optimal solutions (or KKT/Karush-KuhnTucker vectors) to lower-level optimization problems.

Given a single-valued cost mapping $f: X \times Y \rightarrow Z$, a set-valued constraint mapping $S: X \Rightarrow Y$, and a preference $\prec$ with properties (a)-(c) from Definition 3.1, we formulate the multiobjective parametric optimization problem with general constraints as follows:

find a local optimal solution to $f(x, y)$ with respect to $\prec$ subject to $y \in S(x)$, 
where the local optimality of $(\bar{x}, \bar{y}) \in \operatorname{gph} S$ is thus understood in the sense that $f(\bar{x}, \bar{y})$ is not preferred to $f(x, y)$, with respect to the given preference $\prec$ on $Z$, for any feasible point $(x, y) \in \operatorname{gph} S$ close to $(\bar{x}, \bar{y})$.

Our first theorem provides necessary optimality conditions for the multiobjective problem (3.1) in the qualified form ensuring that a dual element (generalized multiplier) associated with the the cost mapping in optimality conditions is nonzero.

Theorem 3.2 (qualified necessary conditions in multiobjective parametric optimization). Let $(\bar{x}, \bar{y})$ be a local optimal solution to the multiobjective problem (3.1), where the preference $\prec$ possesses properties (a)-(c) from Definition 3.1. Assume that the cost mapping $f: X \times Y \rightarrow Z$ between Asplund spaces is continuous around $(\bar{x}, \bar{y})$ and that the constraint mapping $S: X \rightrightarrows Y$ is locally closed-graph around this point. Denote $\dot{\bar{z}}:=f(\bar{x}, \bar{y})$ and impose the following coderivative qualification condition:

$$
\left[\left(x^{*}, y^{*}\right) \in D^{*} f(\bar{x}, \bar{y})(0),-x^{*} \in D^{*} S(\bar{x}, \bar{y})\left(y^{*}\right)\right] \Longrightarrow x^{*}=y^{*}=0 .
$$

Assume also that either $f$ is $S N C$ at $(\bar{x}, \bar{y})$, or $S$ is $S N C$ at this point and the closure of the preference level set $\mathrm{cl} \mathcal{L}: X \Rightarrow Z$ is ISNC at $(\bar{z}, \bar{z})$. Then there exists $z^{*} \neq 0$ satisfying the optimality conditions

$$
0 \in D^{*} f(\bar{x}, \bar{y})\left(z^{*}\right)+N((\bar{x}, \bar{y}) ; \operatorname{gph} S), \quad z^{*} \in N_{+}(\bar{z} ; \operatorname{cl} \mathcal{L}(\bar{z})) .
$$

If in addition $f$ is strictly Lipschitzian at $(\bar{x}, \bar{y})$, then the qualification condition (3.2) is automatic and the optimality conditions (3.3) are equivalent to

$$
0 \in \partial\left\langle z^{*}, f\right\rangle(\bar{x}, \bar{y})+N((\bar{x}, \bar{y}) ; \operatorname{gph} S) \text { with } z^{*} \in N_{+}(\bar{z} ; \operatorname{cl} \mathcal{L}(\bar{z})) \backslash\{0\} .
$$

Proof. Given $(f, \prec, S)$ in the theorem, consider the set-valued mapping $S_{1}: Z \Rightarrow X \times Y \times Z$ and the set $S_{2} \subset X \times Y \times Z$ defined by

$$
S_{1}(z):=\operatorname{gph} S \times \mathcal{L}(z) \text { and } S_{2}:=\operatorname{gph} f .
$$

It follows directly from property (b) of the preference $\prec$ that

$$
(\bar{x}, \bar{y}, \bar{z}) \in S_{1}(\bar{z}) \cap S_{2}
$$

Let us show furthermore that there is a neighborhood $U$ of $(\bar{x}, \bar{y}, \bar{z})$ such that

$$
S_{1}(z) \cap S_{2} \cap U=\emptyset
$$

whenever $z \neq \bar{z}$ is sufficiently close to $\bar{z}$. Assume the contrary and taking an arbitrary neighborhood $U$ of $(\bar{x}, \bar{y}, \bar{x})$, find a point $z \in \mathcal{L}(\bar{z}))$ close to $\bar{z}$ while not equal to the latter by property (a) of Definition 3.1 such that

$$
S_{1}(z) \cap S_{2} \cap U \neq \emptyset \text {. }
$$

Due to the structure of the set $\ddot{S}_{2}=\operatorname{gph} f$ in (3.5), the latter yields the existence of $(x, y)$ near $(\bar{x}, \bar{y})$ satisfying the conditions

$$
z=f(x, y) \text { and }(x, y, z) \in S_{1}(z)=\operatorname{gph} S \times \operatorname{cl} \mathcal{L}(z) .
$$


Hence $y \in S(x)$ and $f(x, y)) \prec f(\bar{x}, \bar{y})$ by property (c) of the preference $\prec$. This clearly contradicts the local optimality of $(\bar{x}, \bar{y})$ in the multiobjective problem (3.1).

The relationships (3.6) and (3.7) mean that the point $(\bar{x}, \bar{y}, \bar{z})$ is locally extremal for the system $\left\{S_{1}, S_{2}\right\}$ at $\bar{z}$ in the sense of [13, Definition 5.64]. Note that the set $S_{2}$ is locally closed and the set-valued mapping $S_{1}(\cdot)$ is locally closed-graph around the points in question due to their constructions in (3:5) and the assumptions made on $f, S$ and $\prec$ in the theorem. Furthermore, the space $X \times Y \times Z$ is Asplund as the product of Asplund spaces. Thus we can apply to $\left\{S_{1}, S_{2}\right\}$ the extended extremal principle from [13, Theorem 5.68] (see also [16, Theorem 4.3]) in the space $X \times Y \times Z$. According to this result, there are

$$
z_{0} \in \bar{z}+\varepsilon \mathbb{B}_{Z},\left(x_{i}, y_{i}, z_{i}\right) \in(\bar{x}, \bar{y}, \bar{z})+\varepsilon \mathbb{B}_{X \times Y \times Z} \text {, and }\left(x_{i}^{*}, y_{i}^{*}, z_{i}^{*}\right) \in X^{*} \times Y^{*} \times Z^{*}
$$

for $i=1,2$ such that $\left(x_{1}, y_{1}\right) \in \operatorname{gph} S, z_{1} \in \operatorname{cl} \mathcal{L}\left(z_{0}\right), z_{2}=f\left(x_{2}, y_{2}\right)$, and

$$
\begin{aligned}
& \left(x_{1}^{*}, y_{1}^{*}, z_{1}^{*}\right) \in \widehat{N}\left(\left(x_{1}, y_{1}, z_{1}\right) ; S_{1}\left(z_{0}\right)\right), \quad\left(x_{2}^{*}, y_{2}^{*}, z_{2}^{*}\right) \in \widehat{N}\left(\left(x_{2}, y_{2}, z_{2}\right) ; S_{2}\right) \\
& \left\|\left(x_{1}^{*}, y_{1}^{*}, z_{1}^{*}\right)+\left(x_{2}^{*}, y_{2}^{*}, z_{2}^{*}\right)\right\| \leq \varepsilon, \quad\left\|\left(x_{1}^{*}, y_{1}^{*}, z_{1}^{*}\right)\right\|+\left(x_{2}^{*}, y_{2}^{*}, z_{2}^{*}\right) \| \geq 1-\varepsilon
\end{aligned}
$$

Taking into account the structure of $\left\{S_{1}, S_{2}\right\}$ in (3.5) and using the product property

$$
\widehat{N}\left(\cdot ; \Omega_{1} \times \Omega_{2}\right)=\widehat{N}\left(\cdot ; \Omega_{1}\right) \times \widehat{N}\left(\cdot ; \Omega_{2}\right)
$$

for Fréchet normals (see [12, Proposition 1.2]), we get from (3.8) that

$$
\left(x_{1}^{*}, y_{1}^{*}\right) \in \widehat{N}\left(\left(x_{1}, y_{1}\right) ; \operatorname{gph} S\right) \text { and } z_{1}^{*} \in \widehat{N}\left(z_{1} ; \operatorname{cl} \mathcal{L}\left(z_{0}\right)\right) \text {. }
$$

To proceed, pick the sequence $\varepsilon:=1 / k$ as $k \rightarrow \infty$ and add the subindex " $k$ " to the corresponding elements above. By construction, we immediately have that

$$
z_{0 k} \rightarrow \bar{z} \text { and }\left(x_{i k}, y_{i k}, z_{i k}\right) \rightarrow(\bar{x}, \bar{y}, \bar{z}) \text { as } k \rightarrow \infty \text { for } i=1,2 \text {. }
$$

Furthermore, by normalization if necessary, we can always suppose that the sequences $\left\{\left(x_{i k}^{*}, y_{i k}^{*}, z_{i k}^{*}\right)\right\} \subset X^{*} \times Y^{*} \times Z^{*}, i=1,2$, are bounded. Therefore, they are sequentially weak* compact in $X^{*} \times Y^{*} \times Z^{*}$ due to the Asplund property of $X \times Y \times Z$; see [21]. Without loss of generality, suppose that

$$
\left(x_{i k}^{*}, y_{i k}^{*}, z_{i k}^{*}\right) \rightarrow\left(x_{i}^{*}, y_{i}^{*}, z_{i}^{*}\right) \in X^{*} \times Y^{*} \times Z^{*} \text { as } k \rightarrow \infty, \quad i=1,2 \text {. }
$$

Passing to the limit in (3.9) as $k \rightarrow \infty$, we have

$$
\left(x_{2}^{*}, y_{2}^{*}, z_{2}^{*}\right)=-\left(x_{1}^{*}, y_{1}^{*}, z_{1}^{*}\right):=\left(x^{*}, y^{*},-z^{*}\right)
$$

while (3.8) and (3.10) yield as $k \rightarrow \infty$ that

$$
\left(x^{*}, y^{*}\right) \in D^{*} f(\bar{x}, \bar{y})\left(z^{*}\right), \quad-x^{*} \in D^{*} S(\bar{x}, \bar{y})\left(y^{*}\right), \text { and } z^{*} \in N_{+}(\tilde{z} ; \operatorname{cl} \mathcal{L}(\bar{z}))
$$

by taking into account the normal and coderivative constructions in (2.1), (2.3), and (2.7). 
Let us show now that $\left(x^{*}, y^{*}, z^{*}\right) \neq 0$ for the limiting elements built above under the SNC/ISNC assumptions imposed in the theorem. To proceed by contradiction, suppose that $\left(x^{*}, y^{*}, z^{*}\right)=0$. Then

$$
\left(x_{1 k}^{*}, y_{1 k}^{*}, z_{1 k}^{*}\right) \stackrel{w^{*}}{\rightarrow} 0 \text { and }\left(x_{2 k}^{*}, y_{2 k}^{*}, z_{2 k}^{*}\right) \stackrel{w^{*}}{\rightarrow} 0 \text { as } k \rightarrow \infty
$$

Assuming that $S$ is SNC at $(\bar{x}, \bar{y})$ and that $\operatorname{cl} \mathcal{L}$ is ISNC at $(\bar{z}, \bar{z})$, we get $\left\|\left(x_{1 k}^{*}, y_{1 k}^{*}, z_{1 k}^{*}\right)\right\| \rightarrow 0$ as $k \rightarrow \infty$, which contradicts the second (nontriviality) relationship in (3.9). On the other hand, if $f$ is assumed to be SNC at $(\bar{x}, \bar{y})$, then (3.12) yields that $\left\|\left(x_{2 k}^{*}, y_{2 k}^{*}, z_{2 k}^{*}\right)\right\| \rightarrow 0$ as $k \rightarrow \infty$, which also contradicts (3.9).

All the conclusions above we reached without imposing the qualification condition (3.2). If we impose it and suppose that $z^{*}=0$, then $\left(x^{*}, y^{*}\right)=0$ due to (3.11) and (3.2), which contradicts the established nontriviality $\left(x^{*}, y^{*}, z^{*}\right) \neq 0$ and thus justifies the qualified optimality conditions (3.3).

It remains to consider the case when $f$ is strictly Lipschitzian at $(\bar{x}, \bar{y})$. In this case, the scalarization formula from [12, Theorem 3.28] ensures that

$$
\left(x^{*}, y^{*}\right) \in \partial\left\langle z^{*}, f\right\rangle(\bar{x}, \bar{y})
$$

and hence (3.3) is equivalent to (3.4). Moreover, $\left(x^{*}, y^{*}\right)=0$ whenever $z^{*}=0$ by the scalarization formula. Thus the qualification condition (3.2) is obviously satisfied in the strict Lipschitzian case. This completes the proof of the theorem.

To employ the general optimality conditions obtained in Theorem 3.2 to multiobjective problems with more specific constraints described by $S(\cdot)$, one needs actually to get an upper estimate of the basic normal cone $N(\bar{x}, \bar{y}) ; \mathrm{gph} S)$ to the graph of the constraint mapping $S(\cdot)$, which is equivalent to computing/estimating the coderivative $D^{*} S(\bar{x}, \bar{y})$. This can done by using the machinery developed in $[12,13]$, where the reader can find a number of results in the latter direction. Before establishing in this way necessary optimality conditions for MOPECs of our main interest, we present a consequence of Theorem 3.2 in the case of conventional parametric constraint systems given by finitely many equalities and inequalities with real-valued functions:

$$
\begin{aligned}
S(x)=\left\{y \in Y \mid \varphi_{i}(x, y) \leq 0,\right. & i=1, \ldots, m, \\
\varphi_{i}(x, y)=0, & i=m+1, \ldots, m+r\},
\end{aligned}
$$

which are typical for problems of nonlinear programming. In the following corollary we consider the case when the functions $\varphi_{i}$ in (3.13) are locally Lipschitzian. For simplicity, we assume that the cost mapping $f$ is also locally Lipschitzian and that the image space $Z$ is finite-dimensional (and thus no SNC condition is needed), although more general assumptions are allowed by Theorem 3.2. Given $(\bar{x}, \bar{y})$, define the active index set

$$
I(\bar{x}, \bar{y}):=\left\{\ddot{\imath} \in\{1, \ldots, m+r\} \mid \varphi_{i}(\bar{x}, \bar{y})=0\right\}
$$

of equality and active inequality constraints. 
Corollary 3.3 (multiobjective optimization problems with equality and inequality constraints). Let $(\bar{x}, \bar{y})$ be a local optimal solution to problem (3.1) with the constraint mapping $S$ given by (3.13). Assume that both spaces $X$ and $Y$ are Asplund while $\operatorname{dim} Z<\infty$, that $f$ and $\varphi_{i}$ are locally Lipschitzian around $(\bar{x}, \bar{y})$, and that the following constraint qualification condition is satisfied:

$$
\left[\sum_{i \in I(\bar{x}, \bar{y})} \lambda_{i} x_{i}^{*}=0\right] \Longrightarrow\left[\lambda_{i}=0, i \in I(\bar{x}, \bar{y})\right]
$$

for any $\lambda_{i} \geq 0$ as $i \in I(\bar{x}, \bar{y})$ and subgradients $x_{i}^{*} \in \partial \varphi_{i}(\bar{x}, \bar{y})$ as $i \in\{1, \ldots, m\} \cap I(\bar{x}, \bar{y})$ and $x_{i}^{*} \in \partial \varphi_{i}(\bar{x}, \bar{y}) \cup \partial\left(-\varphi_{i}\right)(\bar{x}, \bar{y})$ as $i=m+1, \ldots, m+r$. Then there is $z^{*} \in N_{+}(\bar{z}, \mathrm{cl} \mathcal{L}(\bar{z})) \backslash\{0\}$ satisfying the optimality condition

$$
\begin{aligned}
0 & \in \partial\left\langle z^{*}, f\right\rangle(\bar{x}, \bar{y})+\left\{\sum_{i \in\{1, \ldots, m\} \cap I(\bar{x}, \bar{y})} \lambda_{i} \partial \varphi_{i}(\bar{x}, \bar{y})\right. \\
& \left.+\sum_{i=m+1}^{m+r} \lambda_{i}\left(\partial \varphi_{i}(\bar{x}, \bar{y}) \cup \partial\left(-\varphi_{i}\right)(\bar{x}, \bar{y})\right) \mid \lambda_{i} \geq 0 \text { for all } i \in I(\bar{x}, \bar{y})\right\} .
\end{aligned}
$$

Proof. Applying Theorem 3.2, observe first that $f$ is strictly Lipschitzian and SNC at $(\bar{x}, \bar{y})$, since $Z$ if finite-dimensional. Then the optimality condition (3.15) follows from (3.4) due to the upper estimate of the basic normal cone

$$
\begin{aligned}
N((\bar{x}, \bar{y}) ; \operatorname{gph} S) & \subset\left\{\sum_{i \in\{1, \ldots, m\} \cap I(\bar{x}, \bar{y})} \lambda_{i} \partial \varphi_{i}(\bar{x}, \bar{y})\right. \\
& \left.+\sum_{i=m+1}^{m+r} \lambda_{i}\left(\partial \varphi_{i}(\bar{x}, \bar{y}) \cup \partial\left(-\varphi_{i}\right)(\bar{x}, \bar{y})\right) \mid \lambda_{i} \geq 0 \text { for all } i \in I(\bar{x}, \bar{y})\right\}
\end{aligned}
$$

to the graph of the constraint mapping $S$ from (3.13), which is proved under the constraints qualification condition (3.14) in [12, Corollary 4.36].

In the case of multiobjective problems with smooth equality and inequality constraints, when $\varphi_{i}$ are strictly differentiable at $(\bar{x}, \bar{y})$, the qualification condition (3.14) reduces to the classical Mangasarian-Fromovitz constraint qualification in nonlinear programming and the necessary optimality condition (3.15) can be written as

$$
0 \in \partial\left\langle z^{*}, f\right\rangle(\bar{x}, \bar{y})+\sum_{i=1}^{m+r} \lambda_{i} \nabla \varphi_{i}(\bar{x}, \bar{y}) \text { with } z^{*} \in N_{+}((\bar{z} ; \operatorname{cl} \mathcal{L}(\bar{z}))
$$

accompanied by the conventional sign and complementary slackness relations:

$$
\lambda_{i} \geq 0, \lambda_{i} \varphi_{i}(\bar{x}, \bar{y})=0 \text { for } i=1, \ldots, m .
$$

Note that, by $[12$, Theorem 3.86$]$, the SNC property of the constraint mapping $S(\cdot)$ from (3.13) needed in Theorem 3.2 for the case of infinite-dimensional spaces $Z$ always holds under the generalized Mangasarian-Fromovitz constraint qualification (3.14).

Next consider MOPECs defined in (3.1) with equilibrium constraints $y \in S(x)$ described by solution maps to the parameter-dependent generalized equations (1.1). For simplicity, we present qualified necessary conditions for local optimal solutions to such problems when both mappings $f$ and $q$ are strictly Lipschitzian at $(\bar{x}, \bar{y})$. 
Theorem 3.4 (qualified necessary conditions for MOPECs with generalized equation constraints). Let $(\bar{x}, \bar{y})$ be a local optimal solution to the MOPEC defined in (3.1) with the equilibrium constraints $y \in S(x)$ given by

$$
S(x):=\{y \in Y \mid 0 \in q(x, y)+Q(x, y)\},
$$

where $f: X \times Y \rightarrow Z, q: X \times Y \rightarrow P$, and $Q: X \times Y \rightrightarrows P$ are mappings between Asplund spaces and where the preference $\prec$ satisfies the requirements listed in Definition 3.1. Assume that both mappings $f$ and $q$ are strictly Lipschitzian at $(\bar{x}, \bar{y})$ with $\bar{z}:=f(\bar{x}, \bar{y})$ and $\bar{p}:=-q(\bar{x}, \bar{y}) \in Q(\bar{x}, \bar{y})$, that $Q$ is closed-graph around $(\bar{x}, \bar{y}, \bar{p})$, that $\operatorname{cl} \mathcal{L}$ is ISNC at $(\bar{z}, \bar{z})$ (automatic if $\operatorname{dim} Z<\infty$ ), and that the following Fredholm qualification condition is satisfied: the adjoint generalized equation

$$
0 \in \partial\left\langle p^{*}, q\right\rangle(\bar{x}, \bar{y})+D^{*} Q(\bar{x}, \bar{y}, \bar{p})\left(p^{*}\right)
$$

has only the trivial solution $p^{*}=0$. Then there is $z^{*} \neq 0$ such that

$$
0 \in \partial\left\langle z^{*}, f\right\rangle(\bar{x}, \bar{y})+\partial\left\langle p^{*}, q\right\rangle(\bar{x} ; \bar{y})+D^{*} Q(\bar{x}, \bar{y}, \bar{p})\left(p^{*}\right) \text { and } z^{*} \in N_{+}(\bar{z} ; \operatorname{cl} \mathcal{L}(\bar{z}))
$$

with some $p^{*} \in P^{*}$, provided that either $\operatorname{dim} P<\infty$ or $Q$ is $S N C$ at $(\bar{x}, \bar{y}, \bar{p})$.

Proof. Employing Theorem 3.2 in the case of $S$ given by (3.16), we need to check that the assumptions made here ensure the fulfillment of those made in Theorem 3.2 and then to express the necessary optimality condition (3.3) in terms of the initial data of (3.16). This can be done by using the generalized differential and SNC calculi developed in [12].

It follows from [12, Theorem 4.46] and the scalarization formula (2.6) applied to the strictly Lipschitzian mapping $g: X \times Y \rightarrow P$ that

$$
N((\bar{x}, \bar{y}) ; \operatorname{gph} S) \subset\left\{\partial\left\langle p^{*}, q\right\rangle(\bar{x}, \bar{y})+D^{*} Q(\bar{x}, \bar{y}, \bar{p})\left(p^{*}\right) \mid p^{*} \in P^{*}\right\}
$$

provided that the Fredholm qualification condition of this theorem holds and that either $Q$ is SNC at $(\bar{x}, \bar{y} ; \bar{p})$ or $\operatorname{dim} P<\infty$. To justify the SNC property of $S$ in (3.16), observe that the graph of $S$ in (3.16) admits the inverse image representation

$$
\operatorname{gph} S=g^{-1}(\operatorname{gph} Q) \text { with } g(x, y):=(x, y,-q(x, y)) .
$$

Using [12, Theorem 3.84] on the preservation of the SNC property under inverse images, it is not hard to check that the qualification condition of the latter theorem reduces, in the setting (3.20) under consideration, to the afore-mentioned Fredholm qualification condition, while the SNC property of a set under the inverse image in $[12$, Theorem 3.84$]$ is exactly the SNC property of $Q$ at $(\bar{x}, \bar{y}, \bar{p})$. Hence, by [12, Theorem 3.84], $S$ is SNC at $(\bar{x}, \bar{y})$ if the Fredholm qualification condition holds if either $P$ is finite-dimensional, or $Q$ is SNC at $(\bar{x}, \bar{y}, \bar{p})$ and $g$ possesses the so-called partial SNC property. The latter property is automatic for locally Lipschitzian mappings by [12, Corollary 1.69]. Combining all the above and substituting (3.19) into (3.3), we arrive at (3.18) under the assumptions made in theorem.

Observe that Theorem 3.2 potentially gives us another opportunity to derive necessary optimality conditions for the MOPEC under consideration by ensuring the SNC property 
of the cost mapping $f$ without imposing the ISNC requirement on $\mathrm{cl} \mathcal{L}$. However, the strict Lipschitzian assumption imposed on $f$ implies that the image space $Z$ must be of finite dimension; see [12, Corollary 3.30]. Since in this case the level set mapping is obviously ISNC, we do not get any alternative to the assumptions made in theorem.

Note that the name of Fredholm qualification condition coined in this paper is motivated by the analogy with Fredholm's alternative for integral equations, where the triviality of solutions to the adjoint equation is a crucial condition for solvability of the original one. The above Fredholm qualification condition imposing the triviality of solutions to the adjoint generalized equation seems to be of a similar crucial importance for the theory of generalized equations and associated optimization problems with' equilibrium constraints.

Various representations and concretizations of the Fredholm constraint qualification and the optimality condition of Theorem 3.4 can be obtained when the set-valued mapping $Q$ in (3.16) is given in more specific forms associated with remarkable classes of equilibrium constraints. Some of such results for MOPECs are considered in more detail in the subsequent sections. They are mainly related to the corresponding representations and estimations of the coderivative $D^{*} Q$ for multivalued field mappings $Q$ generating equilibrium constraints (1.1). Let us now mention exact explicit calculations of the coderivative $D^{*} Q$ for convexgraph mappings $Q$ obtained in [12, Subsection 4.4.1]. Note that in the latter case the SNC property of $Q$ is closely related to the finite-codimension property of the convex set $\operatorname{gph} Q ;$ see [1] and [12, Subsection 1.1.4] for precise formulations and detailed studies. It is important to observe that if $Q$ in (3.16) is given by

$$
Q(y)= \begin{cases}E & \text { for } y \in \Omega \\ \emptyset & \text { otherwise }\end{cases}
$$

with convex sets $E$ and $\Omega$ and if $q$ is smooth around $(\bar{x}, \bar{y})$, then the above Fredholm constraint qualification reduces to the classical Robinson qualification condition

$$
0 \in \operatorname{int}\left\{q(\bar{x}, \bar{y})+\nabla_{y} q(\bar{x}, \bar{y})(\Omega-\bar{y})+E\right\},
$$

which has been highly recognized in optimization theory and applications.

\section{Subdifferential MOPECs with Composite Potentials}

In this section we study MOPECs with respect to general preference relations defined above subject to equilibrium constraints of type (1.1), where the multivalued parameter-dependent mapping $Q(x, y)$ is represented in the subdifferential form with composite potentials

$$
Q(x, y)=\partial(\psi \circ g)(x, y), \quad(x, y) \in X \times Y,
$$

generated by the composition of a single-valued mapping $g: X \times Y \rightarrow W$ and an extendedreal-valued function $\psi: W \rightarrow \overline{\mathbb{R}}$ acting between Banach spaces. The (first-order) subdifferential in the given description (4.1) is understood in our basic sense (2.4), although other subdifferentials may be used in this scheme as well. 
The composite subdifferential form (4.1) under consideration in (1.1) is typical for many important applications of generalized equations and associated optimization problems with equilibrium constraints. Let us mention the case when $g=I$ (identity mapping) and $\psi(\cdot)=\delta(\cdot ; \Omega)$ is the indicator function of a convex set $\Omega$, which equals 0 on $\Omega$ and $\infty$ otherwise. In this case, relationships (1.1) and (4.1) reduce to the classical variational inequality (1.2). Model (1.1), (4.1) involving parameter-dependent field mappings $Q(x, y)$ allows us to cover also the case of quasivariational inequalities corresponding to.(1.2) with moving convex sets $\Omega=\Omega(x, y)$. Indeed, in this case

$$
Q(x, y)=N(y ; \Omega(x, y))=\partial_{y} \delta(y ; \Omega(x, y))
$$

which can often be written in the composite form (4.1) with a nice mapping $g=g(x, y)$. Furthermore, form (1.1) with the composite subdifferential structure (4.1) is convenient for modeling hemivariational inequalities and their various modifications related to nonconvex functions $\psi$ in $(4.1)$; see $[6,12,20]$ for more discussions and references.

Observe that equilibrium constraints (1.1) with the composite subdifferential structure (4.1) contain by construction a first-order variational information arising, in particular, from first-order necessary conditions in lower-level optimization problems. Thus necessary conditions (and related results) for upper-level problems with equilibrium constraints of the subdifferential type naturally require certain second-order generalized differential objects.

Recall the second-order subdifferential notion for extended-real-valued functions used in what follows; see the book [12] and its references for more details and historical comments. Given $\varphi: X \rightarrow \overline{\mathbb{R}}$ finite at $\widetilde{x}$ and given $\bar{y} \in \partial \varphi(\bar{x})$, the second-order subdifferential of $\varphi$ at $\bar{x}$ relative to $\bar{y}$ is defined by

$$
\partial^{2} \varphi(\bar{x}, \bar{y})(u):=\left(D^{*} \partial \varphi\right)(\bar{x}, \bar{y})(u), \quad u \in X^{* *},
$$

i.e., as the coderivative (2.3) of the first-order subdifferential mapping (2.4). When $\varphi \in C^{2}$ around $\bar{x}$, the set (4.3) is a singleton for each $u \in X^{* *}$ reducing to the classical second-order derivative (Hessian) of $\varphi$ at $\bar{x}$ :

$$
\partial^{2} \varphi(\bar{x})(u)=\left\{\nabla^{2} \varphi(\bar{x})^{*} u\right\}, \quad u \in X^{* *}
$$

where the adjoint operation is not needed for $u \in X$ by the symmetricity of the classical Hessian operator. In general, (4.3) defines a positively homogeneous set-valued mapping from $X^{* *}$ into $X^{*}$, which possesses an extensive calculus in both finite and infinite dimensions; see [12]. Besides various situations and examples considered in the books $[12,13]$ and the references therein, we particularly refer the reader to the papers $[4,14,15]$ containing precise calculations of the second-order subdifferential for favorable classes of extended-realvalued functions arising in constraint optimization and equilibrium problems, as well as in their applications to mechanical and economic modeling.

Let us derive necessary optimality conditions for MOPECs with equilibrium constraints governed by the subdifferential generalized equations with composite potentials

$$
0 \in q(x, y)+\partial(\psi \circ g)(x, y)
$$


where $q: X \times Y \rightarrow X^{*} \times Y^{*}, g: X \times Y \rightarrow W$, and $\psi: W \rightarrow \overline{\mathbb{R}}$. The first theorem concerns in fact the case of $g=g(y)$ in (4.4), i.e., when the multivalued part of (4.4) is parameter-independent. On the other hand, it covers MOPEC models, where some of the spaces may be arbitrary Banach. To proceed in this case, we need to recall the appropriate modifications of the normal and subdifferential (and hence coderivative) constructions from Section 2, which possess the 'required calculus in the general Banach space setting under consideration; see [12]. Actually, the only modification required in the general Banach space setting is that, instead of the sequential Painlevé-Kuratowski outer limits of Fréchet normals and subgradients in (2.1) and (2.4), we now need to consider their $\varepsilon$-enlargements

$$
\begin{aligned}
& \widehat{N}_{\varepsilon}(x ; \Omega):=\left\{x^{*} \in X^{*} \mid \frac{\left\langle x^{*}, u-x\right\rangle}{\|u-x\|} \leq \varepsilon\right\} \text { as } \varepsilon \geq 0 \\
& \widehat{\partial}_{\varepsilon} \varphi(x):=\left\{x^{*} \in X^{*} \mid \frac{\varphi(u)-\varphi(x)-\left\langle x^{*}, u-x\right\rangle}{\|u-x\|} \geq-\varepsilon\right\}
\end{aligned}
$$

respectively, and to include a sequence $\varepsilon_{k} \downarrow 0$ in the limiting process. It is known [12] that one can equivalently reduce (4.5) to (2.2) and (2.5) in the afore-mentioned sequential limiting procedure if the space in question is Asplund and the sets and functions are, respectively, locally closed and lower semicontinuous around the reference points.

As before, we restrict our consideration to MOPECs with strictly Lipschitzian cost mappings. Note that the closed-graph assumption (in the norm topology of $W \times W^{*}$ ) on the subdifferential mapping $\partial \psi$ imposed in the next and subsequent results is automatic if either $\psi$ is locally continuous, or it is amenable (see below) at the points under consideration.

Theorem 4.1 (qualified necessary conditions for subdifferential MOPECs with parameter-independent potentials). Let $\prec$ be a preference on $Z$ satisfying the requirements of Definition 3.1, and let $(\bar{x}, \bar{y})$ be a local optimal solution to the following MOPEC:

$$
\text { minimize } f(x, y) \text { with respect to } \prec
$$

subject to the equilibrium constraint

$$
y \in S(x):=\{y \in Y \mid 0 \in q(x, y)+\partial(\psi \circ g)(y)\},
$$

where $f: X \times Y \rightarrow Z$ is strictly Lipschitzian at $(\bar{x}, \bar{y})$, where $g$ is strictly differentiable at $(\bar{x}, \bar{y})$ with the surjective partial derivative $\nabla_{x} q(\bar{x}, \bar{y})$, where $g=g(y) \in C^{2}$ around $\bar{y}$ with the surjective derivative $\nabla g(\bar{y})$, and where $\operatorname{cl} \mathcal{L}$ is ISNC at $(\bar{z}, \bar{z})$ with $\bar{z}:=f(\bar{x}, \bar{y})$. Assume also that the spaces $X$ and $Z$ are Asplund while $\operatorname{dim} Y<\infty$ and $W$ is Banach, and that the graph of the subdifferential mapping $\partial \psi$ is locally closed around $(\bar{w}, \bar{v})$ with $\bar{w}:=g(\bar{y})$ and with $\bar{v}$ being a unique solution to the system

$$
-q(\bar{x}, \bar{y})=\nabla g(\bar{y})^{*} \bar{v}, \quad \bar{v} \in \partial \psi(\bar{w}) .
$$

Then there are $z^{*} \in N_{+}(\bar{z} ; \mathrm{cl} \mathcal{L}(\bar{z})) \backslash\{0\}$ and $u \in Y$ satisfying

$$
\begin{aligned}
0 \in & \partial\left\langle z^{*}, f\right\rangle(\bar{x}, \bar{y})+\nabla q(\bar{x}, \bar{y})^{*} u \\
& +\left(0, \nabla^{2}\langle\bar{v}, g\rangle(\bar{y})^{*} u+\nabla g(\bar{y})^{*} \partial^{2} \psi(\bar{w}, \bar{v})(\nabla g(\bar{y}) u)\right) .
\end{aligned}
$$


Proof. Employ Theorem 3.4 with $P=Y^{*}\left(=\mathbb{R}^{m}\right), Q(y)=\partial(\psi \circ g)(y)$, and

$$
\partial\langle u, q\rangle(\bar{x}, \bar{y})=\nabla q(\bar{x}, \bar{y})^{*} u, \quad u \in Y,
$$

due to the assumed strict differentiability of $q$ at $(\bar{x}, \bar{y})$. Observe that $Q=\partial(\psi \circ g)$ is locally closed-graph around the reference point by the assumptions on $\partial \psi$ and $g$. Since the partial derivative $\nabla_{x} q(\bar{x}, \bar{y})$ is surjective and $Q=Q(y)$, the Fredholm qualification condition of Theorem 3.4 is clearly satisfied. To express further the first necessary optimality condition in (3.18), we need to compute the coderivative

$$
D^{*} Q(\bar{y}, \bar{p})(u)=\partial^{2}(\psi \circ g)(\bar{y}, \bar{p})(u) \text { with } \bar{p}:=-q(\bar{x}, \bar{y})
$$

which reduces, due to the structure of (4.1) and the definition in (4.3), to computing the second-order subdifferential of the composition involved.

Using the appropriate second-order subdifferential chain rule from [12, Theorem 1.127] held in general Banach spaces under the surjectivity assumption on $\nabla g(\bar{y})$ for the mapping - $g \in C^{2}$, we get the equality

$$
\partial^{2}(\psi \circ g)(\bar{y}, \bar{p})(u)=\nabla^{2}\langle\bar{v}, g\rangle(\bar{y})^{*} u+\nabla g(\bar{y})^{*} \partial^{2} \psi(\bar{w}, \bar{v})(\nabla g(\bar{y}) u)
$$

where $\bar{v}$ is uniquely determined by (4.7). Substituting this into (3.18) with $Q=Q(y)$ and taking into account that $P=Y^{*}$ is finite-dimensional, we arrive at (4.8) under the assumptions made and thus complete the proof of the theorem.

The next result concerns MOPECs governed by parameter-dependent equilibrium constraints in the composite subdifferential form (4.4). In contrast to the preceding theorem, we consider the case when all the spaces involved but the image space $Z$ for the cost mapping are finite-dimensional. At the same time, the structure of the composite potential $\psi \circ g$ is significantly more general than in Theorem 4.1: besides the parameter-dependence, we allow $\nabla g(\bar{x}, \bar{y})$ to be nonsurjective. More precisely, we consider the so-called strongly amenable potentials $\psi \circ g$, where $\psi$ is l.s.c. and convex while $g$ is $C^{2}$ around the reference points under the first-order qualification condition

$$
\partial^{\infty} \psi(\bar{w}) \cap \operatorname{ker} \nabla g(\bar{x}, \bar{y})^{*}=\{0\} \text { with } \tilde{w}:=g(\bar{x}, \bar{y}) ;
$$

see [25] and also [12] for more details concerning this remarkable class of functions largely encountered in finite-dimensional variational analysis and parametric optimization; they are useful, in particular, for the study of quasivariational inequalities (4.2). In (4.9),

$$
\partial \psi(\bar{w}):=\left\{w^{*} \in W^{*} \mid\left(w^{*},-1\right) \in N((\bar{w}, \psi(\bar{w})) ; \operatorname{gph} \psi)\right\}
$$

stands for the singular subdifferential of $\psi$ at $\bar{w}$, which reduces to the singleton $\{0\}$ if $\psi$ is locally Lipschitzian around $\bar{w}$.

Theorem 4.2 (qualified necessary conditions for subdifferential MOPECs with parameter-dependent amenable potentials). Let $\prec$ be a preference on $Z$ satisfying 
the requirements of Definition 3.1 , and let $(\bar{x}, \bar{y})$ be a local optimal solution to the MOPEC (4.6) with the equilibrium constraint

$$
y \in S(x):=\left\{y \in \mathbb{R}^{m} \mid 0 \dot{\epsilon} q(x, y)+\partial(\psi \circ g)(y)\right\}
$$

where $f: \mathbb{R}^{n} \times \mathbb{R}^{m} \rightarrow Z$ is strictly Lipschitzian at $(\bar{x}, \bar{y})$ while $Z$ is Asplund, where $q: \mathbb{R}^{n} \times$ $\mathbb{R}^{m} \rightarrow \mathbb{R}^{n} \times \mathbb{R}^{m}$ is locally Lipschitzian around $(\bar{x}, \bar{y})$, and where the potential $\psi \circ g$ is strongly amenable at this point with $g: \mathbb{R}^{n} \times \mathbb{R}^{m} \rightarrow \mathbb{R}^{l}$. Denote $\bar{z}:=f(\bar{x}, \bar{y}), \bar{w}:=g(\bar{x}, \bar{y})$,

$$
M(\bar{x}, \bar{y}):=\left\{\bar{v} \in \mathbb{R}^{l} \mid \bar{v} \in \partial \psi(\bar{w}), \nabla g(\bar{x}, \bar{y})^{*} \bar{v}=-q(\bar{x}, \bar{y})\right\}
$$

and impose the second-order qualification condition:

$$
\partial^{2} \psi(\bar{w} ; \bar{v})(0) \cap \operatorname{ker} \nabla g(\bar{x}, \bar{y})^{*}=\{0\} \text { for all } \bar{v} \in M(\bar{x}, \bar{y})
$$

and the Fredholm constraint qualification: the adjoint generalized equation

$$
\begin{aligned}
0 \in \partial\langle u, q\rangle(\bar{x}, \bar{y})+ & \bigcup_{\bar{v} \in M(\bar{x}, \bar{y})}\left[\nabla^{2}\langle\bar{v}, g\rangle(\bar{x}, \bar{y})(u)\right. \\
& \left.+\nabla g(\bar{x}, \bar{y})^{*} \partial^{2} \psi(\bar{w}, \bar{v})(\nabla g(\bar{x}, \bar{y}) u)\right]
\end{aligned}
$$

has only the trivial solution $u=0$. Then there are $z^{*} \in N(\bar{z} ; \mathrm{cl} \mathcal{L}(\bar{z})) \backslash\{0\}$ such that

$$
\begin{aligned}
0 \in \partial\left\langle z^{*}, f\right\rangle(\bar{x}, \bar{y})+\partial\langle u, q\rangle(\bar{x}, \bar{y})+ & \bigcup_{\dot{v} \in M(\bar{x}, \bar{y})}\left[\nabla^{2}\langle\bar{v}, g\rangle(\bar{x}, \bar{y})(u)\right. \\
& \left.+\nabla g(\bar{x}, \bar{y})^{*} \partial^{2} \psi(\bar{w}, \bar{v})(\nabla g(\bar{x}, \bar{y}) u)\right]
\end{aligned}
$$

with some $u \in \mathbb{R}^{n} \times \mathbb{R}^{m}$, provided that $\mathcal{L}$ is ISNC at $(\bar{z}, \bar{z})$.

Proof. Based on Theorem 3.4 with $P=\mathbb{R}^{n} \times \mathbb{R}^{m}$ and

$$
Q(x, y)=\partial(\psi \circ g)(x, y)
$$

and taking into account that

$$
D^{*} Q(\bar{x}, \bar{y}, \bar{p})(u)=\partial^{2}(\psi \circ g)(\bar{x}, \bar{y}, \bar{p})(u) \text { with } \bar{p}:=-q(\bar{x}, \bar{y}),
$$

we need to employ an appropriate second-order subdifferential chain rule, which is in fact available for strongly amenable functions under the assumptions made in the theorem involving the second-order qualification condition (4.10); see [12]. Using in this vein [12, Corollary 3.76], we get

$$
\partial^{2}(\psi \circ g)(\bar{x}, \bar{y}, \bar{p})(u) \subset \bigcup_{\bar{v} \in M(\bar{x}, \bar{y})}\left[\nabla^{2}\langle\bar{v}, g\rangle(\bar{x}, \bar{y})(u)+\nabla g(\bar{x}, \bar{y})^{*} \partial^{2} \psi(\bar{w}, \bar{v})(\nabla g(\bar{x}, \bar{y}) u)\right]
$$

for all $u \in \mathbb{R}^{n} \times \mathbb{R}^{m}$. Substituting the latter inclusion into relationships (3.17) and (3.18), we arrive at the the adjoint generalized equation (4.11) and the optimality condition (4.12) for the MOPEC under consideration. 
Observe that the second-order qualification condition (4.10) automatically holds when either $\psi \in C^{1,1}$ around $\bar{w}$ (i.e., it is $C^{1}$ with the local Lipschitzian derivative $\nabla \psi$ ), or the derivative $\nabla g(\tilde{x}, \bar{y})$ is surjective. In general, none of these assumptions is required. We can also see from the proof of the theorem that in the absence of the Fredholm constraint qualification in (4.11), necessary optimality conditions hold in the "non-qualified" form: there are $0 \neq\left(z^{*}, u\right) \in N_{+}(\bar{z} ; \operatorname{cl} \mathcal{L}(\bar{z})) \times\left(\mathbb{R}^{n} \times \mathbb{R}^{m}\right)$ satisfying (4.12).

\section{Subdifferential MOPECs with Composite Fields}

In this concluding section of the paper we study another rather general class of MOPECs subject to equilibrium constraints governed by generalized equations with composite subdifferential fields (1.4), which are described by

$$
0 \in q(x, y)+(\partial \psi \circ g)(x, y)
$$

where $g: X \times Y \rightarrow W, \psi: W \rightarrow \overline{\mathbb{R}}$, and $q: X \times Y \rightarrow W^{*}$. Observe that, in contrast to model (4.4) involving the subdifferential of compositions/potentials, in (5.1) we have compositions of subdifferential mappings with some single-valued mappings in the fields of generalized equations. In particular, model (5.1) describes perturbed implicit complementarity problems of the type: find $y \in Y$ satisfying:

$$
q(x, y) \geq 0, \quad y-g(x, y) \geq 0, \quad\langle q(x, y), y-g(x, y)\rangle=0,
$$

where the inequalities are understood in the sense of some order on $Y$ (e.g., componentwisely in finite-dimensions). Problems of this kind frequently arise in a large spectrum of mathematical models involving various types of economic and mechanical equilibria; see $[6,20]$ and the references therein. First we consider MOPECs with parameter-independent fields in (5.1) and derive necessary optimality conditions in infinite-dimensional settings, which require the general Banach structure of some spaces involved and the Asplund structure of the others. This is done under surjectivity assumptions on the derivatives of the mappings $g$ and $q$ in (5.1) based on the application of Theorem 3.2; note that the usage of Theorem 3.4 in this setting requires more restrictive assumptions on the spaces in question.

Theorem 5.1 (qualified necessary conditions for subdifferential MOPECs with parameter-independent composite fields). Let $(\bar{x}, \bar{y})$ be a local optimal solution to the MOPEC (4.6) with respect to an arbitrary preference $\prec$ satisfying the requirements of Definition 3.1 subject to the equilibrium constraint

$$
y \in S(x):=\{y \in Y \mid 0 \in q(x, y)+(\partial \psi \circ g)(y)\},
$$

where $f: X \times Y \rightarrow Z$ is a mapping between Asplund spaces that is strictly Lipschitzian at $(\bar{x}, \bar{y})$. Assume that $W$ is Banach, that $g: Y \rightarrow W$ is strictly differentiable at $\bar{y}$ with the surjective derivative $\nabla g(\bar{y})$, that $q: X \times Y \rightarrow W^{*}$ is strictly differentiable at $(\bar{x}, \bar{y})$ with the surjective partial derivative $\nabla_{x} q(\bar{x}, \bar{y})$, and that the graph of $\partial \psi$ is locally closed around 
$(\bar{w}, \bar{q})$ with $\bar{w}:=g(\bar{y})$ and $\bar{q}:=-q(\bar{x}, \bar{y})$. Then there is $z^{*} \in N_{+}(\bar{z} ; \operatorname{cl} \mathcal{L}(\bar{z})) \backslash\{0\}$ and $u \in W^{* *}$ satisfying the inclusion

$$
0 \in \partial\left\langle z^{*}, f\right\rangle(\bar{x}, \bar{y})+\nabla q(\bar{x}, \bar{y})^{*} u+\left(0, \nabla g(\bar{y})^{*} \partial^{2} \psi(\bar{w}, \bar{q})(u)\right)
$$

provided that:

(a) either $\operatorname{dim} Z<\infty$,

(b) or $\partial \psi$ is SNC at $(\bar{w}, \bar{q})$ and $\operatorname{cl} \mathcal{L}$ is ISNC at $(\bar{z}, \bar{z})$.

Proof. We employ Theorem 3.2 with $S$ given as the solution map (5.2). By [12, Proposition 4.53] we have the following exact formula for computing the normal cone to the graph of this $S$ in general Banach spaces under the surjectivity assumptions made in theorem:

$$
N((\bar{x}, \bar{y}) ; \operatorname{gph} S)=\bigcup_{u \in W^{* *}}\left[\nabla q(\bar{x}, \bar{y})^{*} u+\left(0, \nabla g(\bar{y})^{*} \partial^{2} \psi(\bar{w}, \bar{q})(u)\right)\right],
$$

where $\bar{w}$ and $\bar{q}$ are defined in the formulation of the theorem. Substituting (5.4) into (3.4), we arrive at the optimality condition (5.3) provided that either property (a) holds (this is equivalent to the SNC property of $f$ due to the strict Lipschitzian assumption made), or the level set map $\operatorname{cl} \mathcal{L}$ is ISNC at $(\bar{z}, \bar{z})$ and the solution map $S$ from (5.2) is SNC at $(\bar{x}, \bar{y})$. Thus we complete the proof of the theorem showing that the SNC property of $S$ at $(\bar{x}, \bar{y})$ is equivalent to the one for $\partial \psi$ at $(\bar{w}, \bar{q})$.

To proceed, observe the inverse mapping representation

$$
\operatorname{gph} S=h^{-1}(\operatorname{gph}(\psi \circ g)) \text { with } h(x, y):=(y,-q(x, y)) .
$$

Clearly, $h$ is strictly differentiable at $(\bar{x}, \bar{y})$ due to this property imposed on $q$ and, moreover, the surjectivity of $\nabla h(\bar{x}, \bar{y})$ is equivalent to the surjectivity of $\nabla_{x} q(\bar{x}, \bar{y})$ assumed in the theorem. By [12, Theorem 1.22], the SNC property of $\operatorname{gph} S$ at $(\bar{x}, \bar{y})$ is equivalent to that of $\operatorname{gph}(\partial \psi \circ g)$ at $(\bar{y}, \bar{q})$. But, since $\nabla g(\bar{y})$ is surjective, the latter is equivalent to the SNC property of $\partial \psi$ at $(\bar{w}, \bar{q})$ due to [12, Theorem 1.74].

In the next theorem we consider subdifferential MOPECs with parameter-dependent composite fields with no surjectivity assumptions on the corresponding derivatives, imposing however more restrictive requirements on the spaces in question.

Theorem 5.2 (qualified necessary conditions for subdifferential MOPECs with parameter-dependent composite fields). Let $(\bar{x}, \bar{y})$ be a local optimal solution to the MOPEC (4.6) with the equilibrium constraint

$$
y \in S(x):=\{y \in Y \mid 0 \in q(x, y)+(\partial \psi \circ g)(x, y)\},
$$

where $f: X \times Y \rightarrow Z$ is a mapping between Asplund spaces that is strictly Lipschitzian at $(\vec{x}, \bar{y})$, where the mappings $q: X \times Y \rightarrow \mathbb{R}^{l}$ and $g: X \times Y \rightarrow \mathbb{R}^{l}$ are locally Lipschitzian around $(\bar{x}, \bar{y})$, and where the graph of $\partial \psi$ is locally closed around $(\bar{w}, \bar{p})$ with $\bar{w}:=g(\bar{x}, \bar{y})$ and $\ddot{p}:=-q(\bar{x}, \bar{y}) ;$ the latter is automatic when $\psi: \mathbb{R}^{l} \rightarrow \overline{\mathbb{R}}$ is either amenable at $\bar{w}$ or continuous around this point. Impose also the second-order qualification condition

$$
\partial^{2} \psi(\bar{w}, \bar{p})(0) \cap\left\{v \in \mathbb{R}^{l} \mid 0 \in \partial\langle v, g\rangle(\bar{x}, \bar{y})\right\}=\{0\}
$$


and the Fredholm constraint qualification: the adjoint generalized equation

$$
0 \in \partial\langle u, q\rangle(\bar{x}, \bar{y})+\left\{\partial\langle v, g\rangle(\bar{x}, \bar{y}) \mid v \in \partial^{2} \psi(\bar{w}, \bar{p})(u)\right\}
$$

has only the trivial solution $u=0$. Then there is $z^{*} \in N(0 ; \Theta) \backslash\{0\}$ satisfying

$$
0 \in \partial\left\langle z^{*}, f\right\rangle(\bar{x}, \bar{y})+\partial\langle u, q\rangle(\bar{x}, \bar{y})+\left\{\partial\langle v, g\rangle(\bar{x}, \bar{y}) \mid v \in \partial^{2} \psi(\bar{w}, \bar{p})(u)\right\}
$$

for some $u \in \mathbb{R}^{l}$, provided that $\operatorname{cl} \mathcal{L}$ is ISNC at $(\bar{z}, \bar{z})$ with $\bar{z}:=f(\bar{x}, \bar{y})$.

Proof. We are now based on Theorem 3.4 with $P=\mathbb{R}^{l}$ and

$$
Q(\dot{x}, y)=(\partial \psi \circ g)(x, y) \text {. }
$$

To apply the Fredholm constraint qualification and necessary optimality condition of this theorem in our setting, we first need to express the coderivative $D^{*}(\partial \psi \circ g)(\bar{x}, \bar{y}, \bar{p})$ of the composition in terms of the corresponding constructions for $\psi$ and $g$. The appropriate coderivative chain rule of $\{12$, Theorem 3.13$]$, the scalarization formula of [12, Theorem 3.28], and construction (4.3) of the second-order subdifferential yield the upper estimate

$$
D^{*}(\partial \psi \circ g)(\bar{x}, \bar{y}, \bar{p})(u) \subset\left\{\partial\langle v, g\rangle(\bar{x}, \bar{y}) \mid v \in \partial^{2} \psi(\bar{w}, \bar{p})(u)\right\}
$$

under the second-order qualification condition (5.6). Substituting (5.9) into (3.17) and (3.18), we arrive at (5.7) and (5.8), and thus complete the proof if the theorem.

If the inner mapping $g$ in the equilibrium constraint composition happens to be strictly differentiable at $(\bar{x}, \bar{y})$, the results of Theorem 5.2 admit significant simplifications.

Corollary 5.3 (qualified necessary conditions for MOPECs with composite fields of special structure). Suppose that in the framework of Theorem 5.2 the inner composite mapping $g: X \times Y \rightarrow \mathbb{R}^{l}$ is strictly differentiable at $(\bar{x}, \bar{y})$. Then all the conclusions of this theorem hold with the replacement of the qualification condition (5.6), the adjoint generalized equation (5.7), and the necessary optimality condition (5.9) by, respectively, those in (4.10),

$$
\begin{gathered}
0 \in \partial\langle u, q\rangle(\bar{x}, \bar{y})+\nabla g(\bar{x}, \bar{y})^{*} \partial^{2} \psi(\bar{w}, \bar{p})(u), \quad \text { and } \\
0 \in \partial\left\langle z^{*}, f\right\rangle(\bar{x}, \bar{y})+\partial\langle u, q\rangle(\bar{x}, \bar{y})+\nabla g(\bar{x}, \bar{y})^{*} \partial^{2} \psi(\bar{w}, \bar{p})(u) .
\end{gathered}
$$

Proof. It simply follows from the subdifferential representation

$$
\partial\langle v, g\rangle(\bar{x}, \bar{y})=\left\{\nabla g(\bar{x}, \bar{y})^{*} v\right\}
$$

held for strictly differentiable mappings.

As mentioned, efficient applications of the qualified necessary optimality conditions for MOPECs derived in Sections 4 and 5 largely depend on computing/estimating (from the above) the second-order subdifferentials $\partial^{2} \psi$ involved in the results obtained. The latter has been done for a number of remarkable classes of extended-real-valued functions that frequently appear in the framework of equilibrium constraints, especially in the context of variational inequalities and complementarity problems; see $[4,12,13,14,15,18,26,27]$ and the references therein. 


\section{References}

[1] Borwein, J.M., Lucet, Y., Mordukhovich, B.S.: Compactly epi-Lipschitzian convex sets and functions in normed spaces. J. Convex Anal. 7, 375-393 (2000)

[2] Borwein, J.M., Strójwas, H.M.: Tangential approximations. Nonlinear Anal. 9, 1347-1366 (1985)

[3] Dempe, S.: Annotated bibliography on bilevel programming and mathematical programs with equilibrium constraints. Optimization 52, 333-359 (2003)

[4] Dontchev, A.L., Rockafellar, R.T.: Characterizations of strong regularity for variational inequalities over polyhedral convex sets. SIAM J. Optim. 7, 1087-1105 (1996)

[5] Fabian, M., Mordukhovich, B.S.: Sequential normal compactness versus topological normal compactness in variational analysis. Nonlinear Anal. 54, 1057-1067 (2003)

[6] Facchinei, F., Pang, J.-S.: Finite-Dimensional Variational Inequalities and Complementarity Problems. Springer Series in Operations Research, Springer, New York, 2003

[7] Fukushima, M., Pang, J.-S.: Quasi-variational inequalities, generalized Nash equilibria, and multi-leader-follower games. Comput. Management Sci. 1, 21-56 (2005)

[8] Hu X., Ralph, D.: Using EPECs to model bilevel games in restructured electricity markets with locational prices. The Judge Institute of Management, University of Cambridge, UK. Preprint (2004)

[9] Jahn, J.: Vector Optimization: Theory, Applications and Extensions. Springer Series in Operations Research, Springer, Berlin, 2004

[10] Luo, Z.Q., Pang, J.-S., Ralph, D.: Mathematical Programs with Equilibrium Constraints. Cambridge University Press, Cambridge, UK, 1996

[11] Mordukhovich, B.S.: Equilibrium problems with equilibrium constraints via multiobjective optimization. Optim. Meth. Soft. 19, 479-492 (2004)

[12] Mordukhovich, B.S.: Variational Analysis and Generalized Differentiation, I: Basic Theory. Grundlehren Series (Fundamental Principles of Mathematical Sciences) 330, Springer, Berlin, 2006

[13] Mordukhovich, B.S.: Variational Analysis and Generalized Differentiation, II: Applications. Grundlehren Series (Fundamental Principles of Mathematical Sciences) 331, Springer, Berlin, 2006

[14] Mordukhovich, B.S., Outrata, J.V.: Second-order subdifferentials and their applications. SIAM J. Optim. 12, 139-169 (2001)

[15] Mordukhovich, B.S., Outrata, J.V., Červinka, M.: Equilibrium problems with complementarity constraints: case study with applications to oligopolistic markets. Optimization, to appear.

[16] Mordukhovich, B.S., Treiman, J.S., Zhu, Q.J.: An extended extremal principle with applications to multiobjective optimization. SIAM J. Optim. 14, 359-379 (2003)

[17] Mordukhovich, B.S., Wang, B.: Generalized differentiation for moving objects. In: 'Optimal Control, Stabilization and Nonsmooth Analysis,' de Queiroz/Malisoff/Wolenski (eds.)., Lecture Notes Cont. Inf. Sci. 301, 351-361, Springer, New York, 2004

[18] Outrata, J.V.: A generalized mathematical program with equilibrium constraints. SIAM J. Control Optim. 38, 1623-1638 (2000) 
[19] Outrata, J.V.: A note on a class of equilibrium problems with equilibrium constraints. Kybernetika 40, 585-594 (2004)

[20] Outrata, J.V., Kočvara, M., Zowe, J.: Nonsmooth Approach to Optimization Problems with Equilibrium Constraints. Kluwer, Dordrecht, The Netherlands, 1998

[21] Phelps, R.R.: Convex Functions, Monotone Operators and Differentiability, 2nd edition. Lecture Notes in Mathematics 1364, Springer, Berlin, 1993

[22] Robinson, S.M.: Generalized equations and their solutions, I: basic theory. Math. Prog. Study 10, 128-141 (1979)

[23] Robinson, S.M.: Strongly regular generalized equations. Math. Oper. Res. 5, 43-62 (1980)

[24] Robinson, S.M.: Variational conditions with smooth constraints: structure and analysis. Math. Prog. 97, 245-265 (2003)

[25] Rockafellar, R.T., Wets, R.J.-B.: Variational Analysis. Grundlehren Series (Fundamental Principles of Mathematical Sciences) 317, Springer, Berlin, 1998

[26] Ye, J.J., Ye, X.Y.: Necessary optimality conditions for optimization problems with variational inequality constraints. Math. Oper. Res. 22, 977-997 (1997)

[27] Ye, J.J., Zhu, Q.J.: Multiobjective optimization problems with variational inequality constraints. Math. Prog. 96, 139-160 (2003) 